\title{
The Diabetic Retinopathy Clinical Research Network (DRCR.net) and Its Contributions to the Treatment of Diabetic Retinopathy
}

\author{
Jennifer K. Sun ${ }^{a, b}$ Lee M. Jampol ${ }^{c}$ \\ a Beetham Eye Institute, Joslin Diabetes Center, Boston, MA, USA; ${ }^{b}$ Department of Ophthalmology, Harvard Medical \\ School, Boston, MA, USA; ${ }^{C}$ Feinberg School of Medicine, Northwestern University, Chicago, IL, USA
}

\section{Keywords}

Diabetic Retinopathy Clinical Research Network .

Proliferative diabetic retinopathy · Panretinal

photocoagulation - Diabetic macular edema

\begin{abstract}
Over the past two decades, the Diabetic Retinopathy Clinical Research Network (now known as the DRCR Retina Network) has contributed to multiple and substantial advances in the clinical care of diabetic eye disease. Network studies helped establish anti-vascular endothelial growth factor (VEGF) agents as an effective alternative to panretinal photocoagulation for eyes with proliferative diabetic retinopathy (PDR) and as first-line therapy for eyes with visual impairment for diabetic macular edema (DME), defined treatment algorithms for the use of intravitreal medications in these conditions, and provided critical data to understand how to better evaluate the diabetic eye using optical coherence tomography and other imaging modalities. Ongoing DRCR.net studies will address whether anti-VEGF therapy is effective at preventing vision-threatening complications in eyes with severe non-proliferative diabetic retinopathy, if photobiomodulation has a beneficial effect in eyes with $\mathrm{DME}$, and whether initiation of DME treatment with bevacizumab and rescue with aflibercept can provide visual outcomes as good as those achieved with aflibercept alone. Future plans for the Network also include the expansion into non-diabetic eye disease in areas such as age-related macular degeneration.
\end{abstract}

(c) 2019 S. Karger AG, Basel

\section{KARGER}

(c) 2019 S. Karger AG, Basel

E-Mail karger@karger.com

www.karger.com/ore

\section{Introduction}

José Cunha-Vaz, MD, $\mathrm{PhD}$, has devoted the last six decades of his life to the study of diabetic retinopathy (DR). From his early days at Moorfields Eye Hospital, to his leadership positions in Portugal and elsewhere in Europe, to his contributions while working at the University of Illinois Eye and Ear Infirmary in Chicago, he has established himself as one of the foremost basic scientists, clinicians, and clinical trialists in the field of DR. One of us (L.M.J.) had an opportunity to work with him during his days at the University of Illinois. He was a serious scientist collaborating with many clinician-scientists, contributing to increased productivity of the entire department. We are delighted to honor him with this review of the work of the Diabetic Retinopathy Clinical Research Network (DRCR.net).

\section{Historical Background}

The Diabetic Retinopathy Study (DRS) was a monumental work, one of the most important clinical trials in the history of medicine. Prior to its initiation and completion, patients with advanced proliferative diabetic retinopathy (PDR) had few choices: hypophysectomy was often done following the demonstration in a patient with Sheehan syndrome and pituitary apoplexy that the severe DR regressed [1]. However, pituitary ablation often re- 
sulted in severe complications, including death from the disruption in the patient's hormonal axis. Experts in the field also noted that when the retina was damaged, for example in patients with retinal scarring from uveitis or optic atrophy, PDR did not develop. As a result, panretinal photocoagulation (PRP) was compared to no intervention in patients with proliferative retinopathy. It was demonstrated that over $90 \%$ of all cases of severe visual loss could be prevented with timely and appropriate PRP [2]. A different form of laser photocoagulation was subsequently proven effective as a treatment for diabetic macular edema (DME), a major cause of vision loss for diabetic patients. The Early Treatment Diabetic Retinopathy Study (ETDRS) demonstrated that focal/grid macular laser photocoagulation, targeting microaneurysms and areas of retinal thickening as well as areas of ischemia could stabilize the progression of DME and decrease vision loss [3]. These two seminal studies changed the treatment of patients with PDR and DME. More recent work by the DRCR.net has complemented the findings of these studies and added new therapeutic choices for clinicians to optimize visual outcomes in patients with diabetes.

The DRCR.net was formed in 2002 through a National Eye Institute and National Institute of Diabetes and Digestive and Kidney Diseases-sponsored cooperative agreement. The objective was to develop a collaborative network to facilitate multicenter clinical research on DR, DME, and associated conditions. Priority initiatives for the new Network were to leverage the combined strengths of academic and community retina sites in the new infrastructure as well as to create opportunities for industry collaboration while maintaining rigorous academic independence from pharmaceutical interests.

The Network's inception came at a critical junction in the evolution of our mechanistic understanding of diabetic eye disease. In 1994, it was first recognized that vascular endothelial growth factor (VEGF) levels in the vitreous and retina were elevated in patients with ocular angiogenesis, including in eyes with PDR [4]. Injection of VEGF into the vitreous of animals resulted in retinopathy resembling severe non-proliferative DR, and also the development of macular edema in primates [5]. Thus, it was a logical step to evaluate intravitreal anti-VEGF injections for the treatment of DME and PDR. In that setting, Network protocols were proposed and implemented to evaluate the efficacy of intravitreal anti-VEGF agents for diabetic eye disease.

Since 2002, the DRCR.net has initiated and completed 30 multicenter studies in DR with over 350 clinical sites enrolled as participating centers over the lifetime of the
Network. These studies have not only helped establish anti-VEGF agents as an effective alternative to PRP for eyes with PDR and as first-line therapy for eyes with visual impairment for DME, but they have also defined treatment algorithms for the use of intravitreal medications in these conditions and provided critical data to better understand how to evaluate the diabetic eye using optical coherence tomography (OCT) and other imaging modalities.

\section{Treatment of DME}

Treatment of DME with macular focal/grid laser stabilized many eyes with clinically significant macular edema and even improved vision in some patients. However, in the ETDRS, a major improvement in vision was not seen in the majority of patients. The realization that VEGF is a key mediator of abnormal vascular permeability in the diabetic eye led to early investigations of antiVEGF therapy for the treatment of DME.

Although pegaptanib sodium was the first anti-VEGF agent developed specifically for intraocular use, it was rapidly superseded by more effective medications of the same class. The anti-VEGF medication bevacizumab had been developed to bind VEGF in cancer patients and, initially, received approval for the indication of metastatic colon cancer. It was subsequently tried as therapy for patients with DME, first systemically, and then intraocularly. An early DRCR.net pilot study demonstrated improvements in retinal thickening and visual acuity in eyes with DME after intravitreal injections of bevacizumab [6]. Bevacizumab was never developed for registration for eye disease but is available for intraocular use off label. An additional therapeutic option became available once Genentech modified the bevacizumab molecule to make it smaller and more likely to penetrate the retina to create ranibizumab.

Protocol I from the DRCR.net was the first definitive phase 3 study to address the efficacy of an intravitreous anti-VEGF agent for treatment of DME. This study compared four treatments for DME: ranibizumab with immediate focal/grid laser, ranibizumab with macular laser given only for persistent DME after 6 months, intraocular triamcinolone plus immediate macular laser, and macular laser with sham injections. This study clearly showed that anti-VEGF given by the protocol-specified prn treatment regimen was highly effective for treatment of DME. Vision improved an average of 8 or 9 letters in the ranibizumab-treated groups as compared to only 3 letters in the 
laser-treated group by the 1-year primary endpoint [7]. In addition, subsequent follow-up of the ranibizumab groups in Protocol I demonstrated sustained improvement in median and mean vision up to five years with a reduced treatment burden down to a median of $0-1$ injection in the 4th and 5th years of follow-up [8]. Studies by pharmaceutical companies confirmed that sustained monthly treatments of anti-VEGF are more effective than laser treatment alone [9-12].

The DRCR.net Protocol I algorithm was the first prn treatment regimen to be developed for anti-VEGF therapy for DME. With slight modifications, this algorithm for treating until stability in thickness and in vision is reached has been the therapy recommended by the DRCR.net in subsequent studies as well. The Network algorithm enables a marked decreased number of injections of ranibizumab after an initial phase of 6 monthly treatments given unless eyes achieve success at the 4 th or 5 th month of follow-up with vision of 20/20 or better and a normal OCT central subfield thickness. Cessation of monthly treatments is allowed once visual acuity and OCT thickness have stabilized over 2 or more visits. Deferral of injections does not require a dry macula or normal vision. Analyses of Network data have demonstrated that when injections are withheld, even eyes with persistent DME frequently maintain good vision over the long-term.

Although bevacizumab and ranibizumab were the first two anti-VEGF medications to be widely used for DME, a third anti-VEGF agent was developed by Regeneron, aflibercept, and as a result, by 2011, there were 3 effective intravitreal anti-VEGF medications being used by the retina community. Bevacizumab was much less expensive than the other two, but relative efficacies were unknown. The DRCR.net Protocol T compared the three medications for DME [13]. On the possibility that one medication might be more effective in eyes with worse vision, possibly associated with higher VEGF levels and more active retinopathy, a pre-specified analysis was planned to compare results from two major subgroups: those with vision of 20/50 or worse and those with vision better than 20/50. The data from Protocol T showed that when the vision was better than 20/50, the efficacy of all three antiVEGF medications for DME was similar. Bevacizumab thinned the retina less than ranibizumab or aflibercept, but the visual acuities were the same up to two years. On the other hand, when baseline vision was 20/50 or worse, there was a superior benefit to aflibercept over the others with statistically significant better vision results at one year. Although in the second year, the margin between the visual gains seen with ranibizumab versus aflibercept

DRCR.net Contributions to DR diminished, an area under the curve analysis showed that aflibercept was superior to the two other medications over a two-year period. The Protocol T treatment algorithm was identical for all three anti-VEGF medications, allowing deferral of injections once eyes achieved sustained stability. In this Protocol, as in Protocol I, a decreasing number of injections was needed in year 2 as compared to year 1 for all 3 treatment groups.

Initial studies of anti-VEGF for DME were all performed in eyes with best corrected visual acuity of 20/32 or worse. However, many clinicians have generalized results from these studies to eyes with milder degrees of visual impairment and routinely treat these eyes with intravitreal anti-VEGF injections. A recent Network study (Protocol $\mathrm{V}$ ) addressed the best therapeutic strategy for eyes with center-involved DME and good vision of 20/25 or better [14]. This study randomized patients to initial management with intravitreal aflibercept versus macular laser treatment versus observation without treatment. Eyes in the laser and observation groups were treated with aflibercept if vision worsened during subsequent follow-up. At two years, the rates of 5 or more letter vision loss were similar in all three groups (16-19\%), and the mean vision in each treatment group was 20/20. Given the costs and potential adverse events associated with intravitreal injections and laser, observation is likely a reasonable initial strategy for treatment-naïve eyes with good vision despite center-involved DME as long as these eyes are followed closely and treated with anti-VEGF if vision worsens.

Studies of animals with diabetic retinal changes and the response of patients with DME to intravitreal corticosteroids indicate that there is an inflammatory component to the development and progression of DR and DME. Intravitreal steroid injections and intravitreal steroid-releasing devices have been shown to have a positive effect in some eyes with DME in terms of decreased DME and increased visual acuity. In Protocol I, although the initial response in terms of visual acuity was almost as good in the steroid-treated group as the anti-VEGF groups within the first 6 months of follow-up, on average steroid-treated eyes had decreasing vision over the next 6 months; and although the average vision improved as some cataracts were removed into the second year, mean vision did not recover to levels comparable to those achieved by the ranibizumab groups. Moreover, phakic eyes given continued intravitreal steroids will almost invariably develop cataract and need cataract surgery. Thus, pseudophakic eyes may be better candidates for intravitreal steroid therapy. Glaucoma is also a well-documented complication of intravitreal steroids. 
Although anti-VEGF therapy is highly effective for most eyes treated with adequate numbers of injections and monthly dosing intervals, approximately $40-50 \%$ of all eyes do not fully respond to anti-VEGF treatment for DME. Thus, intravitreal steroids, while not indicated as first-line therapy for most eyes with DME, have been used by some clinicians for eyes that are incomplete responders to anti-VEGF therapy. In Protocol U, the Network evaluated whether there was a role for steroid as adjuvant therapy in eyes with persistent edema despite anti-VEGF treatment [15]. Patients who had received at least three anti-VEGF injections before entering the study and still had central retinal thickening and decreased vision were given three monthly (additional) injections of ranibizumab to be certain that their response to an anti-VEGF agent was not adequate after 6 injections. At this point, if they still met study eligibility criteria, they were randomized to monthly continued anti-VEGF plus ozurdex (given at baseline and again at 3 months as needed) versus continued anti-VEGF alone. Average gain in visual acuity at 6 months, the primary endpoint, was the same in both groups, although improvement in retinal thickening was greater in the combination steroid/anti-VEGF group. Although this was a phase 2 trial with a relatively small number of patients, it was decided that additional therapeutic studies on this topic were not indicated because of the difficulty in recruiting, and because of the continued improvement of many eyes after six months of treatment with anti-VEGF alone.

\section{Treatment of PDR}

Identification of VEGF as an angiogenic factor led to early investigations that demonstrated efficacy of intravitreal anti-VEGF agents for treatment of diabetic retinal neovascularization. Furthermore, while treating patients with DME, many clinicians noted regression of retinal vasoproliferative changes (PDR). As a result, the decision was made by the DRCR.net to compare an anti-VEGF medication with PRP, which had been the standard care treatment for PDR since it was first proposed in the late 1960s. Although PRP is a highly effective treatment for PDR, which leads to disease quiescence and prevents severe vision loss, it is inherently destructive, resulting in loss of photoreceptors, particularly rods, and thereby leading to loss of peripheral field as well as night vision. Protocol S enrolled patients with PDR who were randomized to receive ranibizumab or PRP [16]. For eyes randomized to PRP, a standardized PRP was done of 1,200-
1,600 spots (using standard laser or approximately 1,800 spots with automated laser). The eyes randomized to ranibizumab received intravitreal injections of ranibizumab on a monthly basis until all neovascularization was regressed or stability had been achieved over at least 2 visits. Patients with visual loss from center-involved DME in either the PRP or ranibizumab groups were treated at baseline with ranibizumab. DME that developed or worsened during follow-up could also be treated with intravitreal ranibizumab.

The results from Protocol S showed that ranibizumab therapy, as utilized in that protocol, was non-inferior to PRP in terms of visual acuity at 2 and 5 years. There was less visual field loss in the patients receiving ranibizumab compared to PRP, but continued field loss was seen in both groups up to 5 years [17]. The patients treated with ranibizumab had less development of new onset DME and less vitrectomy surgery. However, the increased number of visits and greater costs associated with ranibizumab therapy as well as a concern about unchecked disease recurrence and subsequent severe vision loss in noncompliant patients has made the decision whether to use anti-VEGF or PRP as first-line therapy a challenging one for many patients. It is clear that for a patient who is unreliable with follow-up, PRP is often favored since it generally provides long durability of therapeutic effect. If DME is present at the initiation of treatment, then antiVEGF is often the better choice to initiate therapy. Many physicians are now using combination therapy with both PRP and anti-VEGF, which the DRCR.net has not addressed yet in any study.

\section{Evaluation of the Diabetic Eye}

The revolution in care for diabetic eye disease over the last two decades has been greatly informed by advances in retinal imaging that include the development of digital fundus photography, ultrawide field retinal photography and fluorescein angiography (FA), and OCT and angiography. The ETDRS first established 7 standard field stereoscopic fundus photographs as the gold standard method for evaluating DR severity [18]. However, this protocol was time consuming for patients and imagers, required up to 16 flashes of light per eye, and resulted in film slides that could not be readily shared among multiple sites. In 2011, DRCR.net validated the use of digital photography for the clinical research evaluation of the diabetic eye, using a 7 modified-field approach that provided better coverage of the central macula than the ETDRS 7 standard 
fields [19]. In addition, a 4-wide field imaging protocol that incorporates fewer wider-field photographs per eye also provided good agreement for DR severity grading. Most recently, the Network reported that ultrawide field $200^{\circ}$ images masked to show only the idealized ETDRS fields also resulted in largely equivalent DR severity grading when compared to 7 modified field photographs [20]. In contrast, unmasked $200^{\circ}$ images that enable evaluation of the retinal far periphery may increase DR severity level in approximately $10 \%$ of all eyes. An ongoing prospective longitudinal Network study (Protocol AA) will answer the question of whether diabetic lesions in the retinal far periphery also improve our ability to identify eyes at higher risk of future DR worsening independent of baseline ETDRS DR severity level.

Since the pioneering work of Donald Gass, FA has been another important tool in detection and management of DR. It is capable of showing the vascular networks, vascular leakage, capillary and large vessel nonperfusion, the presence of microaneurysms, and other vascular abnormalities. It demonstrates neovascularization of the disc or elsewhere by the pattern of vascular leakage. When the ETDRS classification of levels of DR was developed, it was decided that the incorporation of FA for categorization was not additive to the use of color fundus photographs for assessing future risk of DR worsening. However, FA has been crucial in the care of DR to characterize areas of retinal neovascularization and nonperfusion, and to target leaking microaneurysms and areas of macular nonperfusion in the laser treatment of DME. The role of ultrawide field FA in identifying peripheral nonperfusion and other pathologic changes in the diabetic eye is also being assessed in the ongoing Protocol AA.

With the development of time domain and the spectral domain OCT, much more information is available on which to guide treatment. OCT allows efficient, noninvasive, and reliable cross-sectional imaging of the central retina and optic nerve head. Spectral domain OCT can delineate the thickness of the retina, anatomic changes in the retina including the development of exudation, hyperreflective foci, intraretinal cysts, subretinal fluid, and loss of the normal retinal architecture. The DRCR.net performed initial studies establishing thresholds for normal retinal thickness in diabetic eyes with time domain OCT and spectral domain OCT (Heidelberg, Zeiss, and Optovue). Corresponding thresholds for defining pathologic thickening (DME) have been utilized, in conjunction with visual acuity, to determine treatment need in eyes with DME in the DRCR.net studies. These and other studies have used both changes in central retinal thickness and changes in visual acuity for determining the need for initiation of treatment, need for additional treatment, and for evaluating the efficacy of the treatment. Nonetheless, Network studies have shown a relatively poor correlation between visual acuity and OCT. As described recently, the correlation between retinal thickness and vision as well as changes in these variables is usually in the range of 0.3-0.4. Only about $12-14 \%$ of the variation in change in visual acuity over 2 years is explained by change in OCT thickness in eyes with DME. Despite this, OCT remains an important tool in deciding the efficacy of treatment. The Network has established a change in OCT thickness of $10 \%$ or more as indicative of a real change in thickness that can be considered in the decision to continue or initiate treatment.

Recently, the ability to use OCT angiography (OCT-A) to delineate the perfused retinal vasculature has been added to the DRCR.net protocols. OCT-A, which shows vessels by detecting motion of erythrocytes within the vascular bed, allows visualization of the three main layers of the retinal capillaries. OCT-A shows only perfused vessels with a certain velocity of flow, but it enables imaging of capillary layers that may not be readily seen on FA or color photographs. OCT-A can be better at delineating the capillary layers of the retina, as it is not impeded by the leakage pattern often present in diabetic eyes on FA. Ischemia can be viewed as well. FA requires intravenous administration of the medication, and local and systemic reactions are seen. These are absent with OCT-A. The correlation of OCT-A metrics - such as vessel density with changes in visual acuity, OCT thickness, and color fundus photography (including wide angle) - and ultrawide-field FA findings is being investigated in ongoing DRCR.net protocols.

\section{Summary}

Over the past two decades, the DRCR.net has contributed to multiple and substantial advances in the clinical care of diabetic eye disease, including the understanding that anti-VEGF therapy is the best monotherapy for eyes with DME and is an effective alternative to PRP for treatment of PDR. These protocols have been enabled by the Network's efficient clinical site and coordinating center infrastructure. Ongoing DRCR.net studies will address whether anti-VEGF therapy is effective at preventing vision-threatening complications in eyes with severe nonproliferative DR, whether photobiomodulation has a beneficial effect in eyes with DME, and whether initiation of DME treatment with bevacizumab and rescue with aflibercept can provide visual outcomes as good as those 
achieved with aflibercept alone. In recognition of the Network's role in advancing the care of diabetic eye disease, the National Institutes of Health granted permission in 2017 for the Network (now known as the DRCR Retina Network) to expand into non-diabetic eye disease in areas such as age-related macular degeneration.

\section{Disclosure Statement}

Dr. Sun reports grants from the Jaeb Coordinating Center, National Eye Institute, and Research to Prevent Blindness, as well as non-financial support from Optovue, non-financial support from
Boston Micromachines, grants and non-financial support from Genentech/Roche, grants and non-financial support from Kalvista, non-financial support from Novartis, non-financial support from Novo Nordisk, non-financial support from Adaptive Sensory Technologies, grants and non-financial support from Boehringer Ingelheim, and personal fees from JAMA Ophthalmology, outside the submitted work.

Dr. Jampol reports grants from the National Eye Institute and the Jaeb Coordinating Center, as well as a consultancy with Sanofi.

\section{References}

1 Cogan DG. Diabetic retinopathy. N Engl J Med. 1964 Apr;270(15):787-8.

2 The Diabetic Retinopathy Study Research Group. Photocoagulation treatment of proliferative diabetic retinopathy. Clinical application of Diabetic Retinopathy Study (DRS) findings, DRS Report Number 8. Ophthalmology. 1981 Jul;88(7):583-600.

3 Early Treatment Diabetic Retinopathy Study Research Group. Photocoagulation for diabetic macular edema. Early Treatment Diabetic Retinopathy Study report number 1 . Early Treatment Diabetic Retinopathy Study research group. Arch Ophthalmol. 1985 Dec; 103(12): 1796-806.

4 Aiello LP, Avery RL, Arrigg PG, Keyt BA, Jampel HD, Shah ST, et al. Vascular endothelial growth factor in ocular fluid of patients with diabetic retinopathy and other retinal disorders. N Engl J Med. 1994 Dec;331(22): $1480-7$.

5 Miller JW, Adamis AP, Shima DT, D'Amore PA, Moulton RS, O’Reilly MS, et al. Vascular endothelial growth factor/vascular permeability factor is temporally and spatially correlated with ocular angiogenesis in a primate model. Am J Pathol. 1994 Sep;145(3):574-84.

6 Scott IU, Edwards AR, Beck RW, Bressler NM, Chan CK, Elman MJ, et al.; Diabetic Retinopathy Clinical Research Network. A phase II randomized clinical trial of intravitreal bevacizumab for diabetic macular edema. Ophthalmology. 2007 Oct;114(10):1860-7.

7 Diabetic Retinopathy Clinical Research Network, Elman MJ, Aiello LP, Beck RW, Bressler NM, Bressler SB, Edwards AR, et al. Randomized trial evaluating ranibizumab plus prompt or deferred laser or triamcinolone plus prompt laser for diabetic macular edema. Ophthalmology. 2010 Jun;117(6):1064-77.e35.

8 Elman MJ, Ayala A, Bressler NM, Browning D, Flaxel CJ, Glassman AR, et al.; Diabetic Retinopathy Clinical Research Network. Intravitreal Ranibizumab for diabetic macular edema with prompt versus deferred laser treatment: 5-year randomized trial results. Ophthalmology. 2015 Feb;122(2):375-81.

9 Michaelides M, Kaines A, Hamilton RD, Fraser-Bell S, Rajendram R, Quhill F, et al. A prospective randomized trial of intravitreal bevacizumab or laser therapy in the management of diabetic macular edema (BOLT study) 12-month data: report 2. Ophthalmology. 2010;117(6):1078-86.e2.

10 Mitchell P, Bandello F, Schmidt-Erfurth U, Lang GE, Massin P, Schlingemann RO, et al.; RESTORE study group. The RESTORE study: ranibizumab monotherapy or combined with laser versus laser monotherapy for diabetic macular edema. Ophthalmology. 2011 Apr; 118(4):615-25.

11 Nguyen QD, Brown DM, Marcus DM, Boyer DS, Patel S, Feiner L, et al.; RISE and RIDE Research Group. Ranibizumab for diabetic macular edema: results from 2 phase III randomized trials: RISE and RIDE. Ophthalmology. 2012 Apr;119(4):789-801.

12 Brown DM, Schmidt-Erfurth U, Do DV, Holz FG, Boyer DS, Midena E, et al. Intravitreal aflibercept for diabetic macular edema: 100week results from the VISTA and VIVID studies. Ophthalmology. 2015 Oct;122(10): 2044-52.

13 Wells JA, Glassman AR, Ayala AR, Jampol LM, Bressler NM, Bressler SB, et al.; Diabetic Retinopathy Clinical Research Network. Aflibercept, bevacizumab, or ranibizumab for diabetic macular edema: two-year results from a comparative effectiveness randomized clinical trial. Ophthalmology. 2016 Jun; 123(6):1351-9.

14 Baker CW, Glassman AR, Beaulieu WT, Antoszyk AN, Browning DJ, Chalam KV, et al.; DRCR Retina Network. Effect of initial management with aflibercept vs laser photocoagulation vs observation on vision loss among patients with diabetic macular edema involving the center of the macula and good visual acuity: A randomized clinical trial. JAMA. 2019 May;321(19):1880-94.
15 Maturi RK, Glassman AR, Liu D, Beck RW Bhavsar AR, Bressler NM, et al.; Diabetic Retinopathy Clinical Research Network. Effect of adding dexamethasone to continued ranibizumab treatment in patients with persistent diabetic macular edema: A DRCR Network phase 2 randomized clinical trial. JAMA Ophthalmol. 2018 Jan;136(1):29-38.

16 Gross JG, Glassman AR, Jampol LM, Inusah S, Aiello LP, Antoszyk AN, et al.; Writing Committee for the Diabetic Retinopathy Clinical Research Network. Panretinal Photocoagulation vs Intravitreous Ranibizumab for Proliferative Diabetic Retinopathy: A Randomized Clinical Trial. JAMA. 2015 Nov; 314(20):2137-46.

17 Gross JG, Glassman AR, Liu D, Sun JK, Antoszyk AN, Baker CW, et al.; Diabetic Retinopathy Clinical Research Network. Five-year outcomes of panretinal photocoagulation vs intravitreous ranibizumab for proliferative diabetic retinopathy: A randomized clinical trial. JAMA Ophthalmol. 2018 Oct;136(10): 1138-48.

18 Early Treatment Diabetic Retinopathy Study Research Group. Grading diabetic retinopathy from stereoscopic color fundus photographs an extension of the modified Airlie House classification. ETDRS report number 10. Ophthalmology. 1991 May;98(5 Suppl):786-806.

19 Gangaputra S, Almukhtar T, Glassman AR, Aiello LP, Bressler N, Bressler SB, et al.; Diabetic Retinopathy Clinical Research Network. Comparison of film and digital fundus photographs in eyes of individuals with diabetes mellitus. Invest Ophthalmol Vis Sci. 2011 Aug;52(9):6168-73.

20 Aiello LP, Odia I, Glassman AR, Melia M, Jampol LM, Bressler NM, et al.; Diabetic Retinopathy Clinical Research Network. Comparison of Early Treatment Diabetic Retinopathy Study Standard 7-Field Imaging With Ultrawide-Field Imaging for Determining Severity of Diabetic Retinopathy. JAMA Ophthalmol. 2019 Jan;137(1):65-73. 